\title{
Analytical edge power loss at the lower hybrid resonance: ANTITER IV validation and application to ion cyclotron resonance heating systems
}

\author{
V. Maquet ${ }^{(1,2, \dagger,}$ A. Druart ${ }^{2,3}$ and A. Messiaen ${ }^{1}$ \\ ${ }^{1}$ Laboratory for Plasma Physics - ERM/KMS, Avenue de la Renaissance 30, B-1000 Brussels \\ ${ }^{2}$ Université Libre de Bruxelles, B-1050 Brussels \\ ${ }^{3}$ International Solvay Institutes, CP 231, B-1050 Brussels
}

(Received 26 January 2021; revised 29 October 2021; accepted 1 November 2021)

In the ion cyclotron range of frequency (ICRF), the presence of a lower hybrid (LH) resonance can appear in the edge of a tokamak plasma and lead to deleterious edge power depositions. An analytic formula for these losses is derived in the cold plasma approximation and for a slab geometry using an asymptotic approach and an analytical continuation near the LH resonance. The way to minimize these losses in a large machine like ITER is discussed. An internal verification between the power loss computed with the semi-analytical code ANTITER IV for ion cyclotron resonance heating (ICRH) and the analytic result is performed. This allows us to check the precision of the numerical integration of the singular set of cold plasma wave differential equations. The set of cold plasma equations used is general and can be applied in other parameters domain.

Key words: plasma heating, ICRH, lower hybrid resonance, power loss, edge modes

\section{Introduction}

A potentially important edge power loss mechanism for ion cyclotron resonance heating (ICRH) arising in the presence of a lower hybrid (LH) resonance in the edge of a tokamak plasma has gained renewed interest in view of ICRH application to future large fusion machines like ITER and DEMO as it will likely be present in their scrape-off layer (SOL).

Historically, ion heating at the LH frequency range was expected to take place near the $\mathrm{LH}$ resonance through mode conversion from a fast electromagnetic wave to a slow electrostatic one or through the direct launch of a slow wave (Stix 1965; Hooke \& Bernabei 1972; Bellan \& Porkolab 1974). However, this ion heating scheme proved to be difficult to reproduce and the ion heating efficiency was often too low to be of interest (Porkolab 1984; Gormezano 1986).

In the ion cyclotron range of frequency (ICRF), the same confluence between the fast and the slow waves near the LH resonance can take place in the edge of the plasma where

$†$ Email address for correspondence: Vincent.Maquet@ulb.be 
it can lead to parasitic power losses. The presence of a $\mathrm{LH}$ resonance appears at low densities, in non-inverted heating scenarios (i.e. $f>f_{c i}$ where $f_{c i}$ is the cyclotron frequency of the majority ions and $f$ is the frequency of the radio-frequency (rf) waves launched by the antenna). In these conditions, for a toroidal wavenumber $\left|k_{z}\right|$ which is smaller than the propagation constant in vacuum $k_{0}$, the fast wave undergoes a confluence with the slow wave. On top of this, the misalignment of the Faraday shield (FS) can also create a direct excitation of the slow wave. These two effects can lead to edge power depositions and were discussed theoretically in earlier works (Berro \& Morales 1990; Heikkinen \& Bures 1990; Alava \& Heikkinen 1992; Lawson 1992). Two recent papers (Messiaen \& Maquet 2020; Messiaen, Maquet \& Ongena 2021) provide simple rules to minimize the power loss at this LH resonance, constraining the current distribution on the strap array in amplitude and phase.

Edge power losses in tokamaks should be avoided as they can lead to a reduction of the power deposited in the plasma core and to deleterious impurity release from the first wall of the device. A correlation between ICRH related impurity release and low $\left|k_{z}\right| \lesssim$ $k_{0}$ present in the $k_{z}$ radiation spectrum launched by ICRH antennae was investigated in Maquet \& Messiaen (2020).

Numerically, the LH resonance is particularly hard to simulate (Lu et al. 2016; Nicolopoulos, Campos-Pinto \& Després 2019; Usoltceva et al. 2019; Otin et al. 2020; Tierens et al. 2020). In the edge of a tokamak, the plasma waves can be described in a first approximation by the collisionless cold plasma dielectric tensor. However, at the LH resonance, the rf fields are singular. Numerical computation requires the introduction of finite collisions in the tensor terms and need a very fine spatial resolution to correctly resolve the electromagnetic fields. Moreover, the fast and slow wave modes of the cold plasma coalesce due to their confluence in this region and cannot be treated as separated modes.

A recent upgrade of the semi-analytical code ANTITER II, called ANTITER IV, is now describing the waves launched by an ICRH antenna in the cold plasma limit, including the full description of the fast and slow wave confluence and the LH resonance aspects (Messiaen et al. 2021). The present paper analytically derives the power loss at the LH resonance in $\S 2$, compares the results with the power loss computed numerically by ANTITER IV in $\S 3$ and applies this analysis to an ICRH antenna like the one of ITER in $\S 4$.

\section{Analytical derivation}

The edge plasma is described by the cold dielectric plasma tensor and Maxwell's equations expressed in the radial $x$ direction for a slab geometry and Fourier analysis in the $(y, z)$ directions, where $z$ represents the direction along the total magnetic field $B_{0}$. The plasma wave model considered leads to a system of 4 first-order ordinary differential equations (ODEs) of the form $Y(x)^{\prime}=A(x) Y(x)$

$$
\frac{\mathrm{d}}{\mathrm{d} x}\left(\begin{array}{c}
\mathrm{i} \omega B_{z} \\
E_{y} \\
\mathrm{i} \omega B_{y} \\
E_{z}
\end{array}\right)=\frac{1}{\epsilon_{1}}\left(\begin{array}{cccc}
-k_{y} \epsilon_{2} & k_{0}^{2} \epsilon_{2}^{2}+\left(\epsilon_{1} k_{z}^{2}-k_{0}^{2} \epsilon_{1}^{2}\right) & \epsilon_{2} k_{z} & -k_{y} k_{z} \epsilon_{1} \\
\epsilon_{1}-\frac{k_{y}^{2}}{k_{0}^{2}} & k_{y} \epsilon_{2} & \frac{k_{y} k_{z}}{k_{0}^{2}} & 0 \\
0 & k_{y} k_{z} \epsilon_{1} & 0 & \epsilon_{1}\left(k_{0}^{2} \epsilon_{3}-k_{y}^{2}\right) \\
-\frac{k_{y} k_{z}}{k_{0}^{2}} & k_{z} \epsilon_{2} & \frac{k_{z}^{2}}{k_{0}^{2}}-\epsilon_{1} & 0
\end{array}\right)\left(\begin{array}{c}
\mathrm{i} \omega B_{z} \\
E_{y} \\
\mathrm{i} \omega B_{y} \\
E_{z}
\end{array}\right) .
$$


Moreover,

$$
\begin{gathered}
E_{x}=-\frac{k_{y}}{k_{0}^{2} \epsilon_{1}} \omega B_{z}+\frac{k_{z}}{k_{0}^{2} \epsilon_{1}} \omega B_{y}-i \frac{\epsilon_{2}}{\epsilon_{1}} E_{y}, \\
\omega B_{x}=k_{y} E_{z}-k_{z} E_{y} .
\end{gathered}
$$

In the expressions above $\epsilon_{1}, \epsilon_{2}$ and $\epsilon_{3}$ are the components of the cold dielectric plasma tensor (Swanson 2012) and $\omega$ is the angular frequency of the rf waves launched by the antenna. This set of cold plasma equations is general and can be applied in any parameter domain. In the ICRF, the field components $E_{y}$ and $B_{z}$ can be associated with the fast wave predominant components of interest for heating and $E_{z}$ and $B_{y}$ can be associated with the slow wave predominant components. This system is singular at the location $x_{0}$, where $\epsilon_{1}\left(x_{0}\right)=0$ which corresponds to the LH resonance in the cold plasma description. The resonance location is independent of the wavenumber and only depends on the launched wave frequency and on the plasma composition, density and magnetic field.

In what follows, the method used to derive the power loss at this LH resonance was obtained from an asymptotic expansion of the system of differential equations and from an analytical continuation around the singularity. Analytical continuation has been used for a long time to assess collisionless absorption at cold plasma resonances (e.g. Golant \& Piliya 1972). The application of such method to matrix wave equations is similar to the one used in Faulconer \& Koch (1994) where the authors derive the power loss at the Alfvén resonance.

\subsection{Asymptotic expansion in the vicinity of the $L H$ resonance}

Choosing the position of the LH resonance at the origin $x=0$, one can expand $\epsilon_{1}(x)$ in a Taylor series as $\epsilon_{1}(x)=\epsilon_{1}^{\prime}(0) x+\mathcal{O}\left(x^{2}\right)$, where $\epsilon_{1}^{\prime}$ is the derivative of $\epsilon_{1}$ with respect to $x$. This leads to an asymptotic expression of $A(x)$ of the system (2.1)

$$
A(x)=\frac{A_{0}}{\epsilon_{1}^{\prime} x}+\mathcal{O}\left(x^{0}\right),
$$

with $\epsilon_{1}^{\prime} \equiv \epsilon_{1}^{\prime}(0)$ and where

$$
A_{0} \equiv\left(\begin{array}{cccc}
-k_{y} \epsilon_{2} & k_{0}^{2} \epsilon_{2}^{2} & k_{z} \epsilon_{2} & 0 \\
-\frac{k_{y}^{2}}{k_{0}^{2}} & k_{y} \epsilon_{2} & \frac{k_{y} k_{z}}{k_{0}^{2}} & 0 \\
0 & 0 & 0 & 0 \\
-\frac{k_{y} k_{z}}{k_{0}^{2}} & k_{z} \epsilon_{2} & \frac{k_{z}^{2}}{k_{0}^{2}} & 0
\end{array}\right)
$$

A straightforward computation shows that the matrix $A_{0}$ satisfies

$$
A_{0}^{2}=0 \text {. }
$$

This property will be fundamental in the forthcoming computations. The matrix $A_{0}$ can also be expressed in a way that will become handy later

$$
A_{0}=\frac{1}{k_{0}^{2}}\left(\begin{array}{c}
k_{0}^{2} \epsilon_{2} \\
k_{y} \\
0 \\
k_{z}
\end{array}\right)\left(\begin{array}{llll}
-k_{y} & \epsilon_{2} k_{0}^{2} & k_{z} & 0
\end{array}\right) .
$$




\subsection{Fields near the resonance}

To derive expressions for the tangential $(y, z)$ fields near the resonance we consider $x$ as being a complex variable (i.e. we will consider the analytic continuation of our system of equations over the complex plane). Close to $x=0$, one can truncate the expansion (2.4) as

$$
A(x) \simeq \frac{A_{0}}{\epsilon_{1}^{\prime} x} .
$$

In this approximation, using the property (2.6), we observe that $A(x)$ and its primitive commute. This enables us to use the general theorem derived in appendix A. The solution to the system (2.1) reads

$$
\left(\begin{array}{c}
\mathrm{i} \omega B_{z} \\
E_{y} \\
\mathrm{i} \omega B_{y} \\
E_{z}
\end{array}\right)=\exp \left(\int^{x} A\left(x^{\prime}\right) \mathrm{d} x^{\prime}\right) C=\exp \left(\frac{A_{0}}{\epsilon_{1}^{\prime}} \log (x)\right) C=\left(I+\frac{A_{0}}{\epsilon_{1}^{\prime}} \log (x)\right) C,
$$

where $C \equiv\left(C_{1}, C_{2}, C_{3}, C_{4}\right)^{\mathrm{T}}$ is a constant column vector depending on the initial conditions of the problem. Here, log denotes the principal value of the complex logarithm. The last equality in (2.9) is exact due to the property (2.6). More explicitly, our solution is given by

$$
\left(\begin{array}{c}
\mathrm{i} \omega B_{z} \\
E_{y} \\
\mathrm{i} \omega B_{y} \\
E_{z}
\end{array}\right)=\left(\begin{array}{l}
C_{1} \\
C_{2} \\
C_{3} \\
C_{4}
\end{array}\right)+\alpha(x)\left(\begin{array}{c}
k_{0}^{2} \epsilon_{2} \\
k_{y} \\
0 \\
k_{z}
\end{array}\right)\left(\begin{array}{llll}
-k_{y} & \epsilon_{2} k_{0}^{2} & k_{z} & 0
\end{array}\right)\left(\begin{array}{l}
C_{1} \\
C_{2} \\
C_{3} \\
C_{4}
\end{array}\right),
$$

with $\alpha(x) \equiv \log (x) / k_{0}^{2} \epsilon_{1}^{\prime}$ and where we used relation (2.7).

The expression above clearly shows that all fields except $B_{y}$ are singular at the resonance. One can nevertheless construct a particular combination of them which remains non-singular at $x=0$. Left multiplication of (2.10) by the line vector $\left(\begin{array}{llll}-k_{y} & \epsilon_{2} k_{0}^{2} & k_{0} & 0\end{array}\right)$ leads to

$$
k_{0}^{2} \epsilon_{2} E_{y}-k_{y} \mathrm{i} \omega B_{z}+k_{z} \mathrm{i} \omega B_{y}=k_{0}^{2} \epsilon_{2} C_{2}-k_{y} C_{1}+k_{z} C_{3} \equiv \Upsilon,
$$

where, from (2.2), $\Upsilon$ can be written as

$$
\Upsilon=\mathrm{i} k_{0}^{2} \epsilon_{1} E_{x} .
$$

This indicates a relationship between the fields combination $\Upsilon$ and the radial electric field $E_{x}$. Note that the electric field component $E_{x}$ dominates the other electric field components near the singularity and that the product $\epsilon_{1} E_{x}$, as $\Upsilon$, is non-singular at the LH resonance as was already realized using a field potential approach (Dolgopolov 1966; Golant \& Piliya 1972).

We finally get from (2.10)

$$
\begin{gathered}
\mathrm{i} \omega B_{z}=C_{1}+\alpha(x) \epsilon_{2} k_{0}^{2} \Upsilon, \\
E_{y}=C_{2}+\alpha(x) k_{y} \Upsilon, \\
\mathrm{i} \omega B_{y}=C_{3}, \\
E_{z}=C_{4}+\alpha(x) k_{z} \Upsilon .
\end{gathered}
$$

These are the expressions of the singular part (i.e. up to $\mathcal{O}\left(x^{0}\right)$ corrections) of the $(y, z)$ fields in the vicinity of the LH resonance (located at $x=0$ ). 


\subsection{Power loss at the LH resonance}

Our next goal consists in computing the power loss at the LH resonance using the expressions of the fields derived above. In our case, the power loss $\Delta P$ is given by the difference of the Poynting flux $S(x)$ after and before the resonance

$$
\left.\Delta P \equiv \lim _{\kappa \rightarrow 0^{+}} S(x)\right|_{x=-\kappa} ^{x=+\kappa} .
$$

The Poynting flux can be written as

$$
\begin{aligned}
S(x) & \equiv \operatorname{Re}\left(E_{y} H_{z}^{*}-E_{z} H_{y}^{*}\right) \\
& =\frac{-1}{\omega \mu_{0}} \operatorname{Im}\left[E_{y}\left(\mathrm{i} \omega B_{z}\right)^{*}-E_{z}\left(\mathrm{i} \omega B_{y}\right)^{*}\right] .
\end{aligned}
$$

Defining $a \equiv k_{y} C_{1}, b \equiv k_{0}^{2} \epsilon_{2} C_{2}$ and $c \equiv k_{z} C_{3}$, one has

$$
\begin{gathered}
\operatorname{Im}\left[E_{z}\left(\mathrm{i} \omega B_{y}\right)^{*}\right]=\operatorname{Im}\left(C_{4} C_{3}^{*}\right)+\operatorname{Im}\left[\alpha(x) c^{*} \Upsilon\right] \\
\operatorname{Im}\left[E_{y}\left(\mathrm{i} \omega B_{z}\right)^{*}\right]=\operatorname{Im}\left(C_{2} C_{1}^{*}\right)+\operatorname{Im}\left[\alpha^{*}(x) b \Upsilon^{*}+\alpha(x) a^{*} \Upsilon\right]
\end{gathered}
$$

Making use of these expressions and of the identity $\Upsilon=-a+b+c$, the Poynting flux can be rewritten as

$$
\begin{aligned}
S(x) & =\frac{-1}{\omega \mu_{0}}\left\{\operatorname{Im}\left(C_{2} C_{1}^{*}-C_{4} C_{3}^{*}\right)+\operatorname{Im}\left[\alpha^{*}(x) b \Upsilon^{*}+\alpha(x) a^{*} \Upsilon-\alpha(x) c^{*} \Upsilon\right]\right\} \\
& =\frac{-1}{\omega \mu_{0}} \operatorname{Im}\left(C_{2} C_{1}^{*}-C_{4} C_{3}^{*}\right)+\frac{\operatorname{Im}[\alpha(x)]}{\omega \mu_{0}}|\Upsilon|^{2}
\end{aligned}
$$

We finally have

$$
\Delta P=\left.\lim _{\kappa \rightarrow 0^{+}} S(x)\right|_{x=-\kappa} ^{x=+\kappa}=\frac{|\Upsilon|^{2}}{\omega \mu_{0}}\left(\left.\lim _{\kappa \rightarrow 0^{+}} \operatorname{Im}[\alpha(x)]\right|_{x=-\kappa} ^{x=+\kappa}\right) .
$$

On the other hand, one has the identity

$$
\left.\operatorname{Im}[\alpha(x)]\right|_{x=-\kappa} ^{x=+\kappa} \simeq-\frac{\pi}{k_{0}^{2}\left|\epsilon_{1}^{\prime}\right|} .
$$

A proof of this equality is given in appendix B. Plugging (2.25) into (2.24) leads to the final result

$$
\begin{aligned}
\Delta P & =\frac{-\pi}{k_{0}^{2} \omega \mu_{0}\left|\epsilon_{1}^{\prime}\right|}|\Upsilon|^{2} \\
& =\frac{-\pi k_{0}^{2}}{\omega \mu_{0}\left|\epsilon_{1}^{\prime}\right|}\left|\epsilon_{1} E_{x}\right|^{2},
\end{aligned}
$$

where $E_{x}, \epsilon_{1}$ and $\epsilon_{1}^{\prime}$ are taken at the LH resonance location $x=0$.

This equation confirms that power is lost at the resonance. Relation (2.27) gives insight in the physics underlining the edge power loss: it does not depend on the toroidal electric field $E_{z}$ but on the local radial electric field $E_{x}$. The power loss also inversely depends on 
the derivative of the first dielectric tensor $\epsilon_{1}$ component: a larger density gradient in the edge will lead to lower power losses if the numerator of (2.27) is constant. This formula is the version in SI units of the formula given in Golant \& Piliya (1972) using a local quasi-static assumption. Formula (2.27) avoids this electrostatic assumption but assumes a more stringent structure of the dielectric tensor.

The power loss in relation (2.27) is expressed for a single wavelet $\left(k_{y}, k_{z}\right)$ in the Fourier domain. To find the total power loss corresponding to a strap current distribution of an antenna array, relation (2.27) has to be integrated over the Fourier domain (summed over the considered $\left(k_{z}, k_{y}\right)$ grid in the periodic geometry of a tokamak) with the corresponding spectrum of $E_{x}\left(k_{z}, k_{y}\right)$. From Parseval's theorem, the edge power loss at the LH resonance is obtained

$$
P_{\text {edge }}=\frac{1}{(2 \pi)^{2}} \frac{-\pi \omega \epsilon_{0}}{\left|\epsilon_{1}^{\prime}\right|} \iint\left|\epsilon_{1} E_{x}\right|^{2} \mathrm{~d} k_{y} \mathrm{~d} k_{z},
$$

where $E_{x}, \epsilon_{1}$ and $\epsilon_{1}^{\prime}$ are, again, taken at the lower hybrid resonance location $x=0$.

The power loss relation at the LH resonance derived in (2.27) does not depend on the working range of frequencies considered. However, the rest of the text will be focused on the ion cyclotron range of frequencies. As relation (2.27) is exact, it provides an opportunity to cross-check the numerical integration performed in ANTITER IV at the $\mathrm{LH}$ resonance and will be examined in $\S 3$.

\subsection{Parallelism with previous works}

In the paper of Faulconer \& Koch (1994), the same method as the one presented above is used to derive the losses of the fast wave at the Alfvén resonance. This fast wave is described by a system of 2 first-order ODEs

$$
\frac{\mathrm{d}}{\mathrm{d} x}\left(\begin{array}{c}
\mathrm{i} \omega B_{z} \\
E_{y}
\end{array}\right)=\frac{1}{u}\left(\begin{array}{cc}
-\epsilon_{2} k_{0}^{2} k_{y} & -u^{2}+\epsilon_{2}^{2} k_{0}^{4} \\
u-k_{y}^{2} & \epsilon_{2} k_{0}^{2} k_{y}
\end{array}\right)\left(\begin{array}{c}
\mathrm{i} \omega B_{z} \\
E_{y}
\end{array}\right) .
$$

Here, the Alfvén resonance corresponds to the singular point $u=0$ where $u$ is defined as

$$
u \equiv k_{0}^{2} \epsilon_{1}-k_{z}^{2}
$$

The authors find that the power loss at the Alfvén resonance follows

$$
\Delta P=\frac{-\pi}{\omega \mu_{0}\left|u^{\prime}\right|}\left|u E_{x}\right|^{2}
$$

where $E_{x}, \epsilon_{1}$ and $\epsilon_{1}^{\prime}$ are taken at the LH resonance location $u=0$. Despite the identical form of the two formulas (2.26) and (2.31), one can expect differences in the power loss results. Indeed, the Alfvén resonance is an approximation (Stix 1992; Bellan 1994) in the limit $m_{e} \rightarrow 0$ ) and corresponds to the fast wave power lost at the confluence with the slow wave while the LH resonance corresponds to the power lost by the slow wave through mode conversion.

\section{ANTITER IV}

In this section, the analytical results derived in $\S 2$ are used in the restricted case of ICRH to monitor the precision of the integration performed in ANTITER IV while crossing the LH resonance and to validate the power loss computed. ANTITER is a semi-analytic code describing an antenna in front of a plasma in a plane geometry in the cold plasma limit. The code uses Fourier analysis in the previously defined $(y, z)$ directions and numerical 


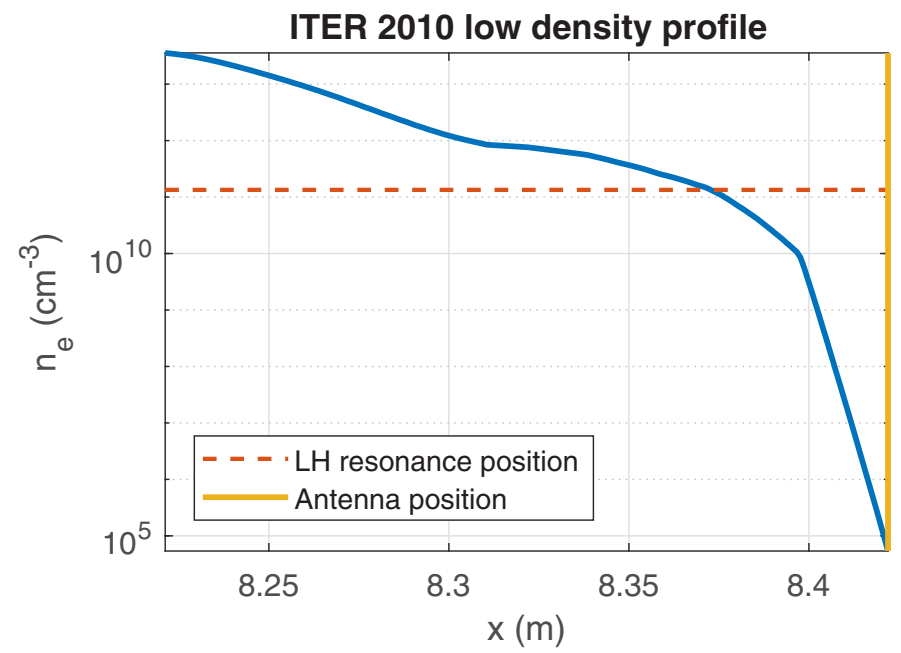

FIGURE 1. ITER 2010 low electron density profile. The antenna and the LH resonance density are displayed.

integration in the radial $x$ one. Concerning the boundary conditions, the tangential fields are matched with the antenna excitation at the antenna aperture plane where an ideal FS adjusts the field polarization. On the plasma core, no reflection, i.e. single-pass absorption, is assumed (Messiaen et al. 2010). The antenna layout is described by a set of boxes recessed into a metal wall containing infinitely thin straps.

The edge plasma electron density profile used for the study is an ITER H-mode reference profile considered as one of the most conservative modelling cases for ICRH coupling (2010low - Carpentier et al. 2011). This specific choice of density profile is used to illustrate the LH power loss taking place in large machines. The electron density profile is presented in figure 1 along with the antenna position and the LH density layer. The ITER magnetic field, minor and major radius used are respectively $B_{0}=5.3 \mathrm{~T}, R_{0}=$ $6.2 \mathrm{~m}, a=2 \mathrm{~m}$. The antenna radial position is $x=8.422 \mathrm{~m}$. The wave frequency used is $55 \mathrm{MHz}$ and the plasma composition chosen is a $0.56-0.44$ deuterium-tritium mix. The periodicity of ITER is approximated through $\left(k_{z, n} k_{y, m}\right)$ Fourier spectral components. For ITER, these Fourier spectral components form a dense set of points and are thus presented as a continuous set in the rest of the text. The size of the grid considered is $-30 \mathrm{~m}^{-1}<k_{z}<30 \mathrm{~m}^{-1}$ and $-25 \mathrm{~m}^{-1}<k_{y}<25 \mathrm{~m}^{-1}$.

ANTITER II only describes the fast wave using the system (2.31), with the approximation of vanishing electron mass. The power transfer from fast wave and slow wave at their confluence is evaluated by the power loss at the Alfvén resonance. ANTITER IV extends the above model to include a detailed description of both the slow and fast waves' excitation and their interaction in an non-homogeneous plasma by solving the system (2.1) (Messiaen et al. 2021). The novelty brought by ANTITER IV lies in its ability to describe direct $E_{z}$ excitation at the antenna, which was impossible in ANTITER II. In the ICRH non-inverted scenario, the following properties are obtained from the dispersion relation of the homogeneous plasma: (i) without direct $E_{z}$ excitation, the slow wave is generated by the confluence with the fast wave which mainly occurs for $\left|k_{z}\right|<k_{0}$; (ii) with a direct $E_{z}$ excitation by the antenna, the slow wave can be excited when $\left|k_{z}\right|>k_{0}$ and can propagate between the cutoff, occurring for $\epsilon_{3}=0$ close to the wall, and the LH layer. 

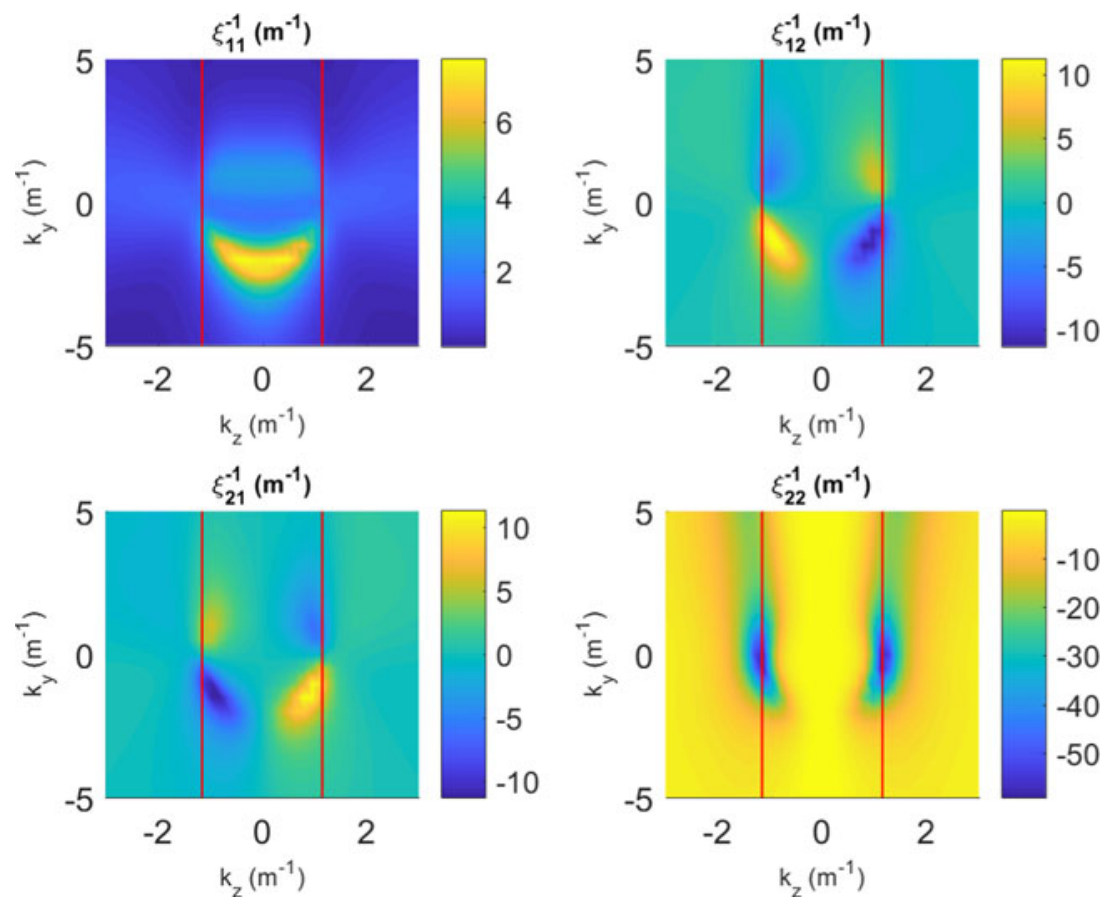

FIGURE 2. The four plasma conductance matrix elements seen at the antenna position as a function of low $\left(k_{y}, k_{z}\right)$. The $k_{z}= \pm k_{0}$ line is also represented by red lines.

The boundary condition at the antenna output is described by four admittance matrix elements $\xi_{i j}^{-1}$ obtained from the integration of (2.1). Those elements take the form

$$
\left(\begin{array}{l}
\omega B_{z} \\
\omega B_{y}
\end{array}\right)=\left(\begin{array}{ll}
\xi_{11}^{-1} & \xi_{12}^{-1} \\
\xi_{21}^{-1} & \xi_{22}^{-1}
\end{array}\right)\left(\begin{array}{l}
E_{y} \\
E_{z}
\end{array}\right)
$$

and describe the relationship between the four tangential plasma components $E_{y}, E_{z}, B_{z}$ and $B_{y}$ for a pair $\left(k_{y}, k_{z}\right)$ considered in the Fourier expansion. The real part of these four admittance matrix elements, the conductance elements, found at the antenna position is presented in the figure 2 for all wavenumbers $\left(k_{y}, k_{z}\right)$ considered and is linked to the power coupled at the antenna aperture $P_{\text {rad }}$ by relations (2.19) and (3.1) as

$$
2 P_{\text {rad }}=\frac{1}{(2 \pi)^{2} \omega \mu_{0}} \operatorname{Re}\left\{\iint\left[\left(\frac{\left|E_{y}\right|^{2}}{\xi_{11}^{*}}+\frac{E_{y} E_{z}^{*}}{\xi_{12}^{*}}\right)-\left(\frac{E_{z} E_{y}^{*}}{\xi_{21}^{*}}+\frac{\left|E_{z}\right|^{2}}{\xi_{22}^{*}}\right)\right] \mathrm{d} k_{y} \mathrm{~d} k_{z}\right\} .
$$

In ANTITER IV, the LH resonance is handled by adding an imaginary part to the dielectric tensor component $\epsilon_{1}$ in order to integrate the system (2.1). This corresponds to the numerical implementation of an analytical continuation. The imaginary part is introduced in the form of collisions in the cold plasma tensor terms (e.g. Swanson (2012), chap. 3, p. 105). The value of this dissipative term is discussed in the next sub-section.

\subsection{Fields near the LH resonance with ANTITER IV}

We first investigate the fields' behaviour near the LH resonance. The fields near the resonance depend on the imaginary dissipative term applied in ANTITER IV. Relation 
(2.11) and appendix B give some intuitions of this dependence: $\epsilon_{1} E_{x}$ being non-singular near the LH resonance, one can write in this region

$$
\left|E_{x}\right| \approx \frac{|C|}{\sqrt{\left(\Delta r \epsilon_{1}^{\prime}\right)^{2}+\operatorname{Im}\left(\epsilon_{1}\right)^{2}}},
$$

with $C$ a constant. The dissipative term replaces the field singularity $E_{x}$ at the resonance by a peak of amplitude $|C| / \operatorname{Im}\left(\epsilon_{1}\right)$ and a $3 \mathrm{~dB}$ width below maximum $w_{3 \mathrm{~dB}}=2 \operatorname{Im}\left(\epsilon_{1}\right) / \epsilon_{1}^{\prime}$ around that resonance. This dissipative term only influences significantly the fields and the power loss over several widths $w_{3} \mathrm{~dB}$ and does not need to be added elsewhere.

An optimal dissipative term and an optimal radial division of the domain exist in ANTITER IV and arise as a trade-off between the numerical cost of the integration and the expected accuracy of the numerical results:

- The optimal step size $\Delta r$ imposed should be smaller than the characteristic peak width $w_{3} \mathrm{~dB}$ of the fields near the resonance in order to correctly resolve their variation. Practically, the integration step size is locally refined near the resonance to $\Delta r$ of 0.1 to $0.02 w_{3} \mathrm{~dB}$. This adaptation can be performed with an automatic adaptive Runge-Kutta routine or based on the $\epsilon_{1}$ value.

- The result of the numerical integration follows the theoretical correction term of (B 8) derived in appendix B which is proportional to $\arctan \left(\operatorname{Im}\left(\epsilon_{1}\right) /\left(\kappa \epsilon_{1}^{\prime}\right)\right) ; \operatorname{Im}\left(\epsilon_{1}\right)$ being small, the numerical accuracy evolves linearly with the dissipative term. For the antenna excitation case considered, convergence can be reached for the full grid for values of $w_{3 \mathrm{~dB}}<10^{-4} \mathrm{~m}$. An example of the obtained agreement is given in the next section, figure 4 .

To illustrate the discussion above, the Poynting flux and the absolute radial electric field $\left|E_{x}\right|$ are displayed in figures $3(a)$ and $3(b)$ for two dissipative terms and a pair $\left(k_{y}, k_{z}\right)$. The dissipative term used are such that the $3 \mathrm{~dB}$ width $w_{3} \mathrm{~dB}$ is equal $10^{-4} \mathrm{~m}$ and $10^{-6} \mathrm{~m}$ which corresponds respectively to a collision frequency $v / \omega$ of $12 \times 10^{-4}$ and $12 \times 10^{-6}$. Figure $3(a)$ shows that two dissipative terms lead to the same Poynting flux jump. Figure $3(b)$ illustrates the asymptotic behaviour of the radial electric field $E_{x}$ when low collisionality is imposed.

For the rest of the paper, unless specified otherwise, the optimal dissipative term leading to $w_{3 \mathrm{~dB}}=10^{-4} \mathrm{~m}$ is chosen as it leads to reliable results with a smaller subdivision of the radial domain.

\subsection{Power loss at the LH resonance with ANTITER IV}

The power losses at the LH resonance using the Poynting flux (2.19) and the analytical power loss (2.27) computed with the fields of ANTITER IV are compared.

This exercise is first performed for a purely poloidal excitation (i.e. $E_{y}\left(k_{y}, k_{z}\right)=1$ at the antenna aperture while ensuring $\left.E_{z}\left(k_{y}, k_{z}\right)=0\right)$. For this specific excitation, the power loss is mainly limited to wavenumbers smaller than the wave propagation constant in vacuum $\left|k_{z}\right|<k_{0}$ as they correspond to the fast wave undergoing a wave confluence with the slow wave. This fact is verified in figure 4. We also observe that the error between the analytical Poynting flux given in (2.27) and the numerical integration of ANTITER IV is negligible. In this case, figure 4 also illustrates the link between the power loss computed and the admittance matrix element as only the first matrix element $\xi_{11}^{-1}$ will enter in the power derivation (3.2).

The same test can be performed for a purely toroidal polarization (i.e. $E_{z}\left(k_{y}, k_{z}\right)=1$ and $\left.E_{y}\left(k_{y}, k_{z}\right)=0\right)$. Here, we see a strong interaction which is no longer limited to the region 

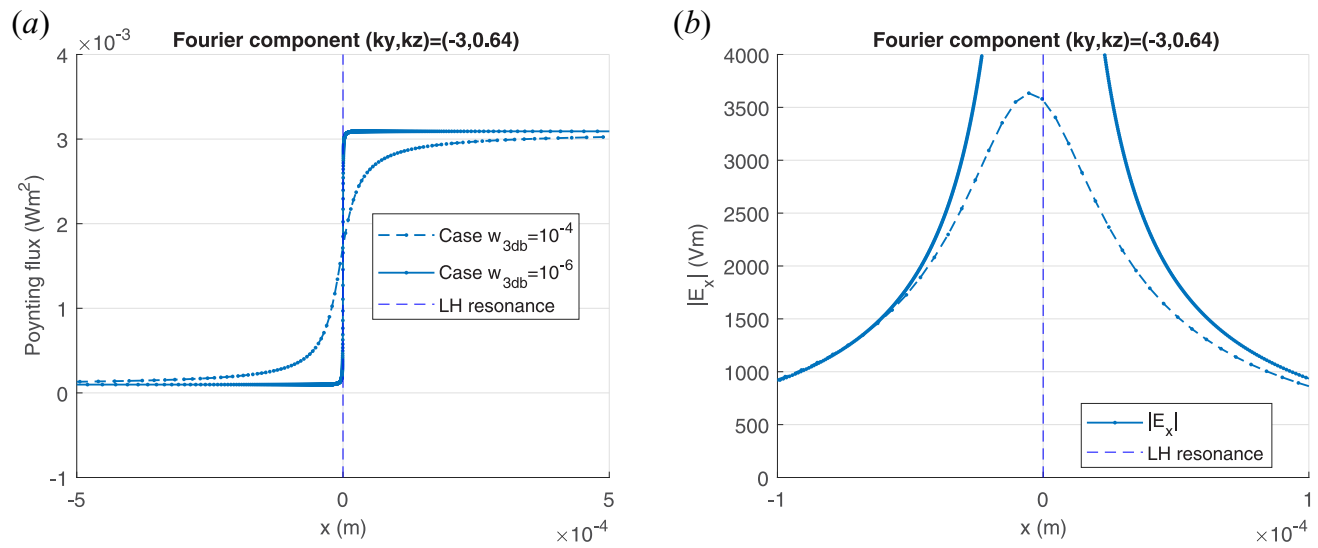

FIgURE 3. (a) Poynting flux jump observed around the LH resonance for a pair $\left(k_{y}, k_{z}\right)$ and for two dissipative terms. (b) Absolute value of the radial electric field $\left|E_{x}\right|$ around the LH resonance for a pair $\left(k_{y}, k_{z}\right)$ and for the same dissipative terms for a pure poloidal electric field excitation.
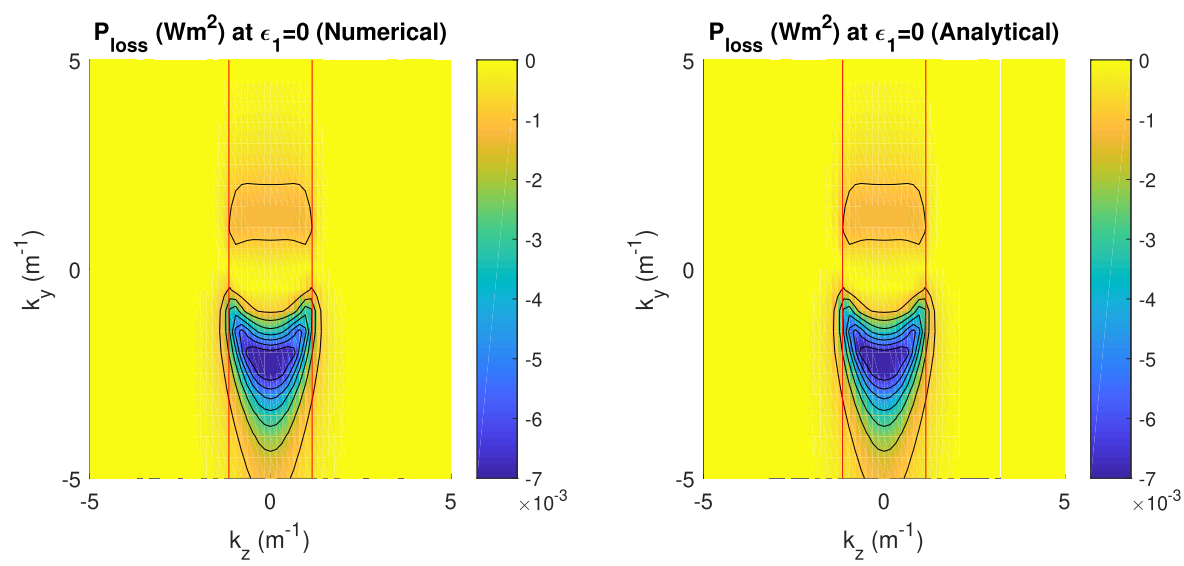

FIGURE 4. Power loss at the LH resonance for a pure $E_{y}\left(k_{z}, k_{y}\right)$ excitation. Red lines delimit the $\left|k_{z}\right|<k_{0}$. Here $k_{0}=1.15 \mathrm{~m}^{-1}$.

$\left|k_{z}\right|<k_{0}$ but extends to $\left|k_{z}\right|>k_{0} \mathrm{~m}^{-1}$ and peaks at $\left|k_{z}\right|=k_{0}$ and $k_{y}$ close to 0 . There is again only a negligible difference between the results obtained with the two methods. The comparison between figure 5 and $\xi_{22}^{-1}$ in figure 2 illustrates the link between the power loss computed due to direct slow wave excitation and the admittance matrix element $\xi_{22}^{-1}$.

For a field-aligned antenna with a field-aligned FS, figure 4 shows that the power losses can be minimized by avoiding the $\left|k_{z}\right|<k_{0}$ region in the power spectrum. The antenna power spectrum can be easily modified by shaping the $E_{y}$ spectrum excited by the antenna (i.e. by the adjustment of the current amplitude and phase distribution over the straps; see $\S 4)$. A FS that is not aligned with the background magnetic field will excite a spurious $E_{z}$ spectrum that can, in turn, lead to non-negligible new losses for low $\left|k_{z}\right|$ above $k_{0}$, as shown in figure 5. Indeed, for an excitation of $E_{y}$ and $E_{z}$ of equal amplitude, the losses due to $E_{z}$ are one order of magnitude larger than the losses due to $E_{y}$. The same magnitude difference is also visible in the conductance matrix elements $\operatorname{Re}\left(\xi_{11}^{-1}\right)$ and $\operatorname{Re}\left(\xi_{22}^{-1}\right)$ shown in figure 2 . Note that wave reflection on metallic elements protruding in the far SOL region and not 

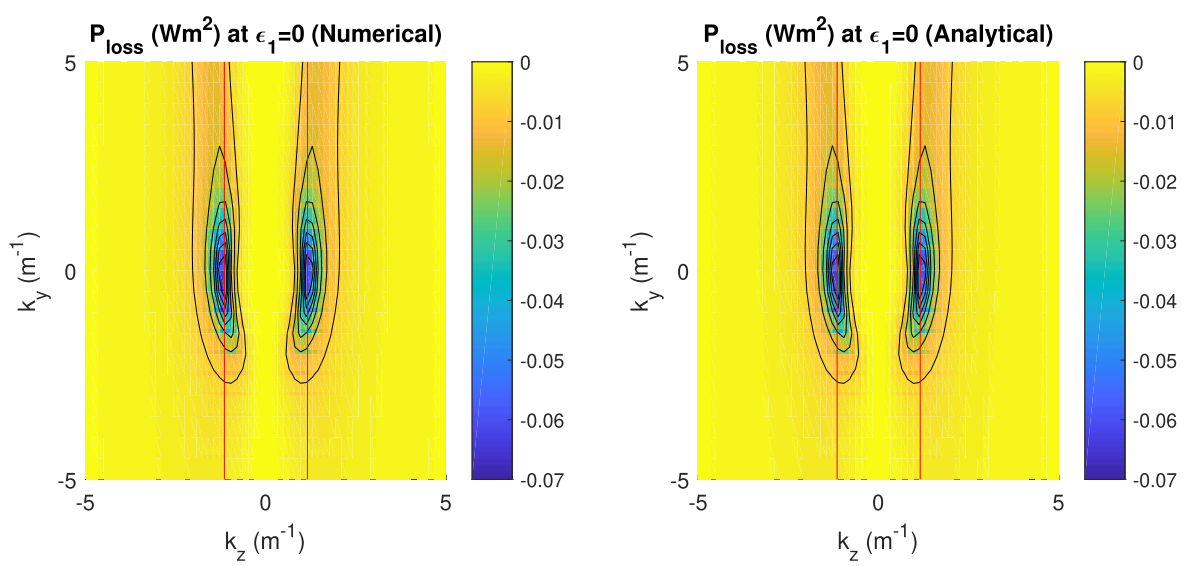

FIgURE 5. Power loss at the LH resonance for a pure $E_{z}\left(k_{z}, k_{y}\right)$ excitation. Red lines delimit the $\left|k_{z}\right|<k_{0}$. Here $k_{0}=1.15 \mathrm{~m}^{-1}$.

parallel to $B_{0}$ will also create an undesirable $E_{z}$ excitation (Kohno, Myra \& D'Ippolito 2015) that cannot be modelled in ANTITER IV. These new losses can be reduced by further depleting the low $\left|k_{z}\right|$ part of the power spectrum at the expense of a reduction in the power coupled to the plasma core (Messiaen et al. 2021).

Finally, one could minimize the power losses by controlling the density profile i.e. by enlarging the density gradient near the LH location while avoiding a similar increase in the numerator of (2.27). This could be done using appropriate gas puff (Zhang et al. 2019) and could lead to a substantial decrease of the ratio of edge power losses to the power coupled to the core by increasing the power coupled to the bulk plasma and decreasing the edge power losses.

\section{Application to an antenna of the ITER type}

The results of the previous sections indicate how to minimize power losses in the presence of a $\mathrm{LH}$ resonance in the plasma edge for a given plasma profile. In this section, ANTITER IV uses an antenna like the one of ITER in front of the ITER 2010low electron density profile to illustrate the conclusions and observations made in $\S \S 2$ and 3 . The antenna model used in ANTITER IV is presented in the figure 6(a) and is composed of 24 straps grouped into triplets (Lamalle et al. 2013). The antenna triplets are illustrated in figure $6(b)$. We first show the effect on the antenna power spectrum of different misalignment of the antenna box and FS with the background magnetic field $B_{0}$. The link between the radial electric field $E_{x}$ and edge power loss is illustrated. Finally, an example of the sensitivity of the edge power loss under different edge density distances and gradients is given.

\subsection{Effect of the antenna box and FS misalignment}

The effect of a misalignment of the antenna box and the FS with the background magnetic field $B_{0}$ was already discussed in Messiaen et al. (2021) and is performed here considering three cases to illustrate the discussion of $\S 3$ with concrete examples. Each case presents the antenna power spectrum for the two conventional phasings $(0 \pi \pi 0)$ and $(0 \pi 0 \pi)$ and a phasing $(0.00,2.9,3.7,0.3)$ minimizing the low $\left|k_{z}\right|$ part of the power spectrum (Maquet $\&$ Messiaen 2020) for a fixed and even current amplitude of 1 A on the straps. The phasing $(0 \pi 0 \pi)$ produces no excitation at $k_{z}=0$ and is rather depleted of low $\left|k_{z}\right|$ excitation due 
(a)

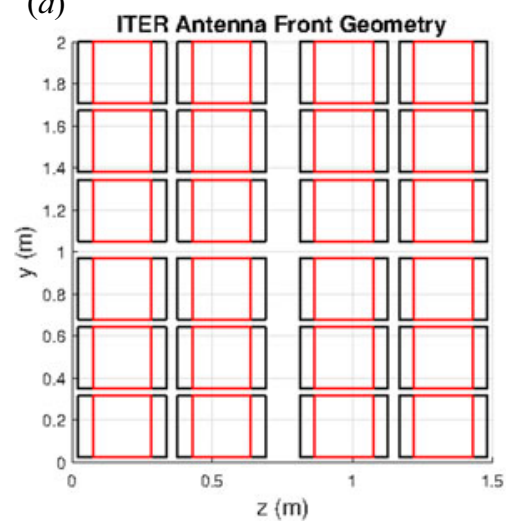

(b)

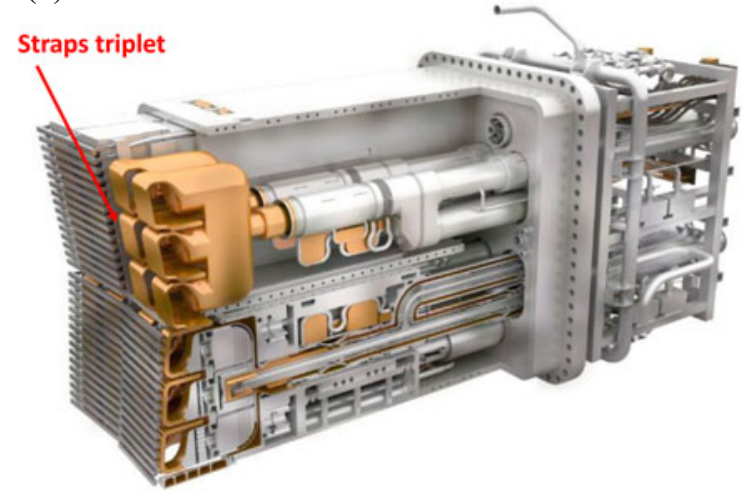

FIGURE 6. (a) Front face of the ITER antenna model used in ANTITER IV. (b) ITER 2012 ICRF antenna design showing the 24 strap array grouped in 8 triplets with their feeding a quarter of the array is shown with the Faraday screen removed (Lamalle et al. 2013).
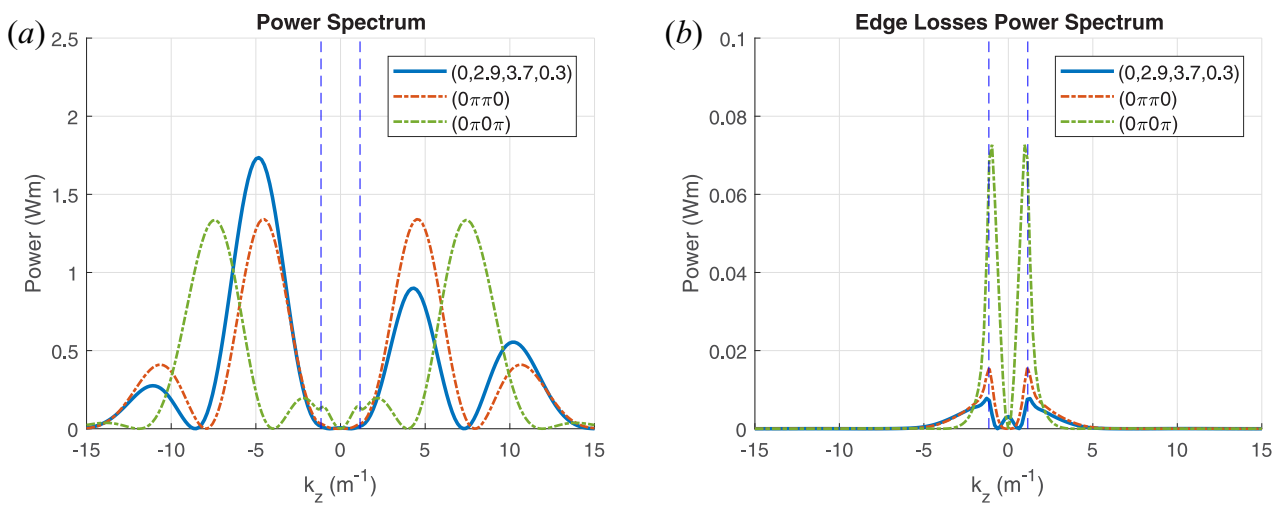

FIgURE 7. An antenna box and a FS aligned to the magnetic field. (a) The $k_{z}$ power spectrum and $(b) k_{z}$ edge power loss spectrum for a current distribution on straps of $1 \mathrm{~A}$ and three different toroidal phasings. A poloidal phasing of $\pi / 2$ is imposed for load resilience. The toroidal phasing $(0.0,2.9,3.7,0.3) \mathrm{rad}$ minimizes the edge LH power losses.

the large $k_{z}$ dominant peak excited in its power spectrum as seen on figure 7 . The phasing $(0 \pi \pi 0)$ shows a larger depletion of excitation at low $\left|k_{z}\right|$ due to the fact that both the excitation and its derivative with respect to $k_{z}$ are cancelled for $k_{z}=0$. Note that the same phasing has allowed high power ICRH in the all metal AUG tokamak (Bobkov et al. 2016). The third phasing minimizes the low $k_{z}$ part of the power spectrum by a simplex method varying the phase current distribution of the strap array. The examples shown below aim at illustrating the effects of the polarization of the antenna excitation and present the expected behaviour for an ITER-like antenna system.

The three cases considered are:

- An antenna box and a FS aligned to the magnetic field. As explained in $\S 3.2$, this case only creates edge losses in the lower $\left|k_{z}\right|<k_{0}$ part of the power spectrum. Figure $7(a)$ presents the power spectrum found for the three cases discussed above. 

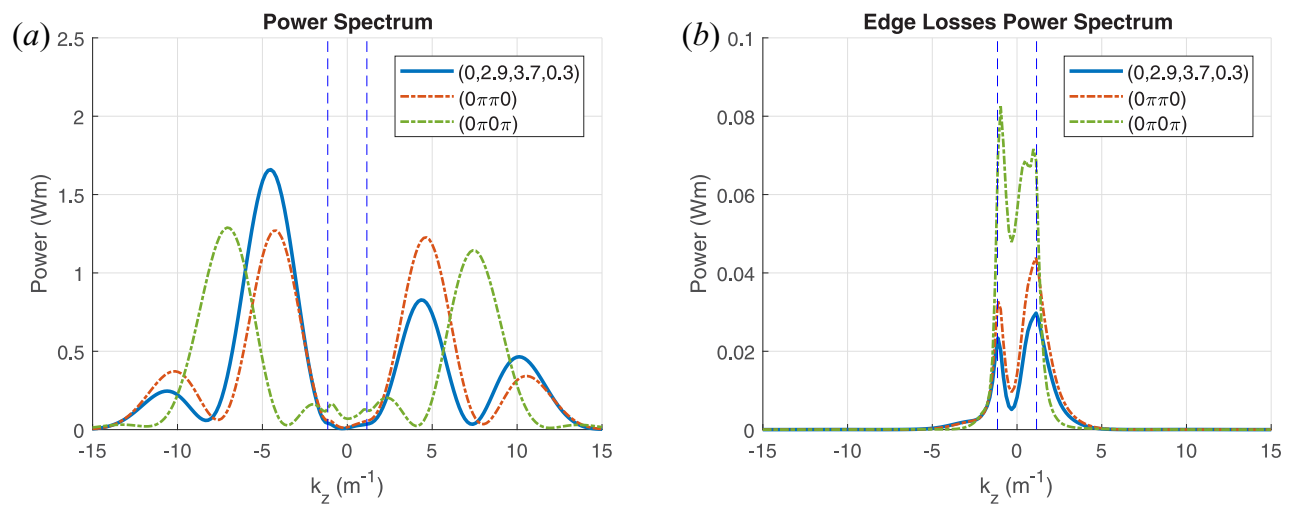

FIGURE 8. A misaligned antenna box and an aligned FS. ( $a$ ) The $k_{z}$ power spectrum and $(b)$ $k_{z}$ edge power loss spectrum for a current distribution on straps of $1 \mathrm{~A}$ and three different toroidal phasings. A poloidal phasing of $\pi / 2$ is imposed for load resilience. The toroidal phasing $(0.0,2.9,3.7,0.3) \mathrm{rad}$ minimizes the edge LH power losses.
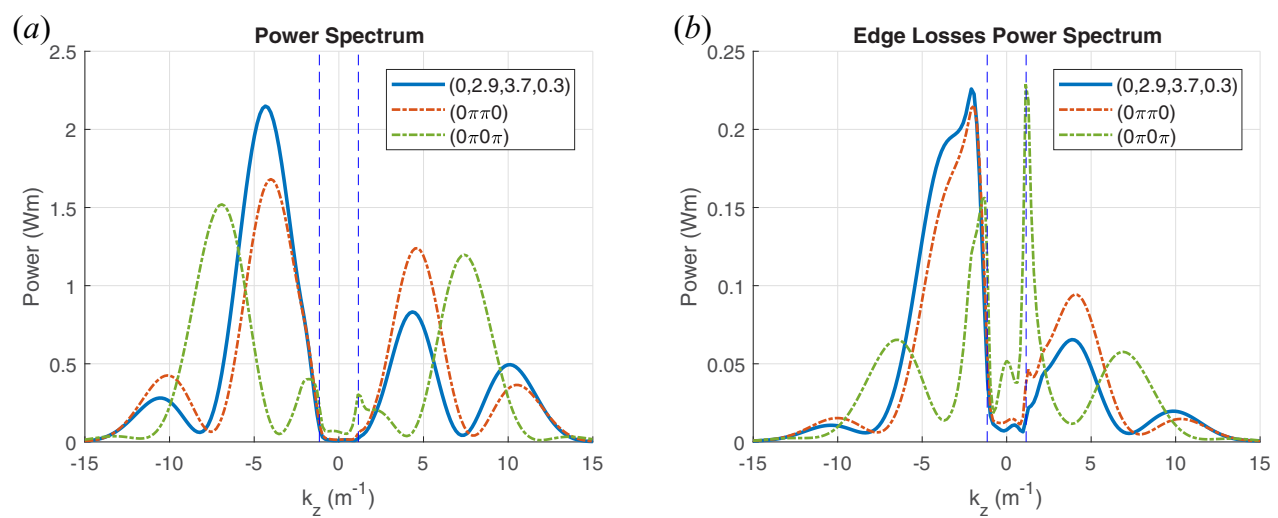

FIGURE 9. A misaligned antenna box and a misaligned FS. (a) The $k_{z}$ power spectrum and (b) $k_{z}$ edge power loss spectrum for a current distribution on straps of $1 \mathrm{~A}$ and three different toroidal phasings. A poloidal phasing of $\pi / 2$ is imposed for load resilience. The toroidal phasing $(0.0,2.9,3.7,0.3) \mathrm{rad}$ minimizes the edge LH power losses.

Figure $7(b)$ presents the related edge power loss spectrum. The figure shows that the LH losses are concentrated at $\left|k_{z}\right|$ smaller or near $k_{0}$ as expected.

- A misaligned antenna box and an aligned FS. This case deforms the power loss spectrum but does not lead to direct spurious $E_{z}$ excitation and consequently only slightly changes the power loss at the LH resonance. The same computation done in figure 7 is presented in figure 8 but with an antenna box tilting at an angle of $15^{\circ}$.

- A misaligned antenna box and a misaligned FS. This case creates an undesirable $E_{z}$ excitation and increases substantially the power loss at the LH resonance as expected from the discussion of $\$ 3.2$. The misalignment of the FS with the magnetic field can be treated in ANTITER IV using the poloidal electric field $E_{y}$ computed in the aligned case and rotating it by an angle of $15^{\circ}$ with respect to $B_{0}$ (Messiaen et al. 2021). This condition corresponds to FS aligned with the triplets array. The result is presented in figure 9. 
(a) Percentage of $P_{\text {loss }}$ (W) in the edge for $(0,2.9,3.8,0.4)$

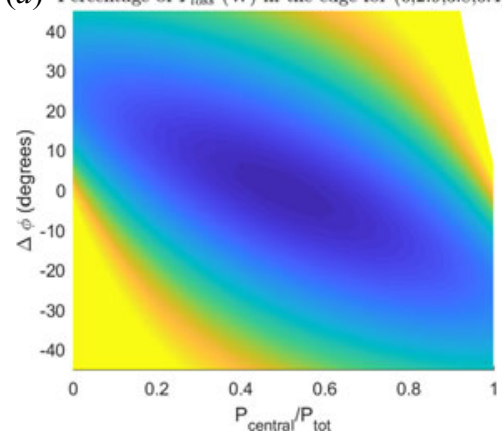

(b) $\iint\left|E_{x}\right|^{2} d k_{y} d k_{z}\left(\mathrm{~V}^{2} \mathrm{~m}^{2}\right)$ at LH resonance for $(0,2.9,3.8,0.4)$
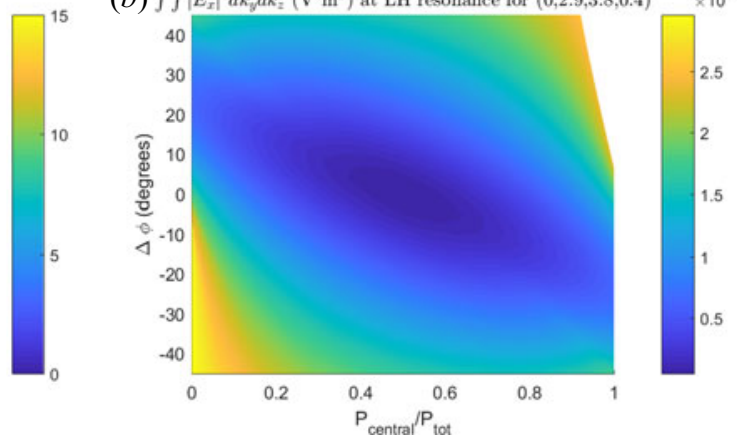

FIGURE 10. Map showing respectively: $(a)$ the percentage of power loss in the edge and $(b)$ the integrated radial electric field $\iint\left|E_{x}\right|^{2} \mathrm{~d} k_{y} \mathrm{~d} k_{z}$ at the LH resonance as a function of the power ratio $P_{\text {central }} / P_{\text {tot }}$ and a central phase deviation $\Delta \phi$ from the phasing minimizing the edge power loss $(0,2.9+\Delta \phi, 3.7+\Delta \phi, 0.3)$.

\subsection{Link with the radial electric field}

One can also verify that the minimization of the LH power loss corresponds to the minimization of the local radial electric field $E_{x}$ at the LH resonance in ANTITER IV. To do so, a scan of the radial electric field is performed as a function of the toroidal power ratio $P_{\text {central }} / P_{\text {tot }}$, between the two inner strap array powers $P_{\text {central }}$ and the total strap array power $P_{t o t}$, and as a function of a phase deviation $\Delta \phi$ to the phasing minimizing the $\mathrm{LH}$ power losses $(0,2.9+\Delta \phi, 3.7+\Delta \phi, 0.3)$ rad found for an antenna box and a FS aligned to the magnetic field. The figure of merit chosen for the radial electric field is its integrated value $\iint\left|E_{x}\right|^{2} \mathrm{~d} k_{y} \mathrm{~d} k_{z}$ computed for a fixed value of collisions $v / \omega$ at the LH position. The strap power is found from the impedance matrix $Z$ computed in ANTITER IV. This matrix relates the voltage and currents at the ports $V=Z I$ and gives access to the power fed at each port $i$ using $P_{i}=\frac{1}{2} V_{i} I_{i}^{*}$.

The result, displayed in figure 10, shows that the minimum of LH power losses corresponds to a minimum of $E_{x}$ excitation at the resonance. The same minimum of power loss corresponds to the minimum of excitation of the low $k_{z}$ part of the power spectrum. It should be noted that the amplitude value of the $E_{x}$ fields at the LH depends on the collision frequency used during the integration and does not have a physical basis.

\subsection{Influence of the edge gradient}

The power loss given by (2.26) is inversely proportional to the derivative of $\epsilon_{1}$ and, consequently, to the gradient of the density profile along the radial direction $x$. To illustrate this, a steepening of the 2010low density profile is enforced by imposing an exponential decay from a point in the profile to the antenna mouth for the misaligned antenna box and FS case discussed in $\S 4.1$. To ensure a nearly constant antenna power coupling, the starting point of the density decay is chosen before the wave cutoff densities of the two toroidal phasing cases $(0 \pi 0 \pi)$ and $(0 \pi \pi 0)$ considered. Indeed, the density profile below the cutoff density has only a weak influence on the coupling (Messiaen \& Weynants 2011). The different density profiles constructed are presented in figure 11(a) along with the LH resonance and the cutoff density values of the 2 phasing cases considered. To ensure convergence in the smallest decay length cases, a smaller optimal dissipative term leading to $w_{3 \mathrm{~dB}}=10^{-5} \mathrm{~m}$ had to be enforced. The corresponding ratio of edge power loss $P_{\text {edge }} / P_{\text {tot }}$ as a function of the decay length imposed is presented in figure $11(b)$ for the 

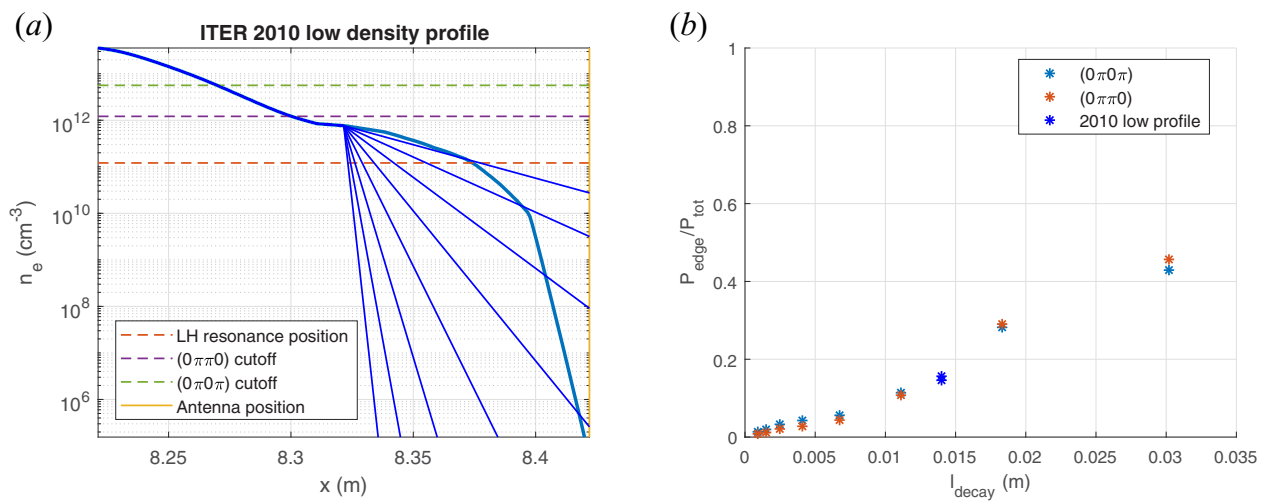

FIGURE 11. (a) Density profile constructed with exponential decaying lengths considered. (b) Corresponding increase of edge power losses as a function of the decay length considered and an even current strap excitation.
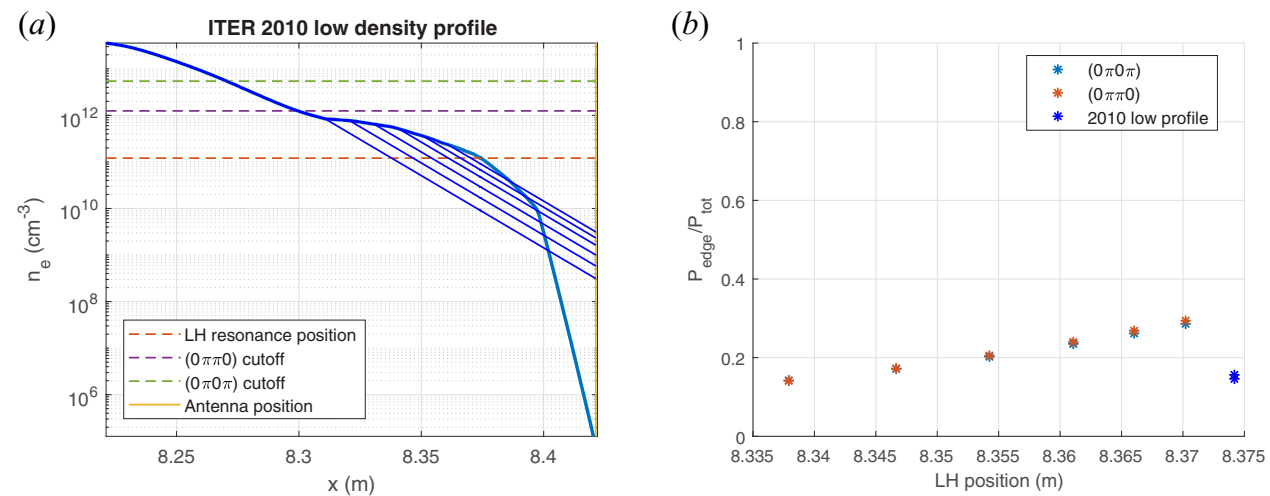

FIGURE 12. (a) Density profile constructed with the same exponential decaying lengths crossing the LH resonance at different position. (b) Corresponding increase of edge power losses as a function of the LH position considered and an even current strap excitation.

conventional toroidal phasings $(0 \pi 0 \pi)$ and $(0 \pi \pi 0)$ and for a even current distribution on straps of $1 \mathrm{~A}$. The same figure also shows the edge power ratio corresponding to the 2010low density profile with an associated decay length best fitting the $\epsilon_{1}$ gradient at the $\mathrm{LH}$ resonance. The increase of power loss with the decrease of the edge density gradient is verified because the numerator of (2.27) does not change much over the $\left(k_{y}, k_{z}\right)$ grid considered.

\subsection{Influence of LH distance}

The sensitivity of the edge power losses to the position of the LH resonance can also be illustrated. This is done in the same condition as $\S 4.3$ by imposing a fixed exponentially decaying density profile at different position from the antenna aperture. The density profiles constructed are presented in figure 12(a) along with the LH resonance and the cutoff density layers of the conventional toroidal phasings $(0 \pi 0 \pi)$ and $(0 \pi \pi 0)$. The corresponding edge power loss ratio $P_{\text {edge }} / P_{\text {tot }}$ as a function of the LH position imposed is potted in figure $12(b)$ for the toroidal phasings $(0 \pi 0 \pi)$ and $(0 \pi \pi 0)$ and for an even current distribution on straps of $1 \mathrm{~A}$. One observes that the edge power loss at the LH resonance 
is relatively independent of its position in this particular case where the numerator (2.27) does not change much over the $\left(k_{y}, k_{z}\right)$ grid considered.

\section{Conclusion}

This paper presents an analytical derivation of the power loss arising in the presence of the singular point $\epsilon_{1}=0$ in the differential system of (2.1) describing an inhomogeneous cold plasma in a slab geometry. The power loss expression found (2.27) is valid for any cold plasma parameter domain considered $\left(f, B_{0}, n_{e}\right.$, ion mix) and can therefore be applied on a large $\left(k_{y}, k_{z}\right)$ region to study the losses arising in any frequency domain of a cold plasma.

We limit our study to the ICRH power loss arising for a non-inverted scenario in the presence of a $\mathrm{LH}$ resonance located in the plasma edge of a fusion machine. The analysis first shows that, while the radial electric field $E_{x}$ and the cold plasma tensor component $\epsilon_{1}$ are singular at the LH resonance, their product remains finite. The power loss found (2.27) is linked to the local product $\left|\epsilon_{1} E_{x}\right|$ at the LH resonance position and is inversely proportional to the slope along the radial direction $x$ of the cold dielectric tensor component $\epsilon_{1}$ at the LH location. The analytical formula of the power loss is then used to verify the accuracy of the numerical integration performed by the semi-analytical code ANTITER IV using the same slab description and cold plasma model. Excellent agreement is found between the two. Finally, we illustrate possible scenarios that could minimize the ITER ICRH power losses:

(i) In a screen aligned to the total magnetic field $B_{0}$ scenario, one should avoid the excitation of the lower $\left|k_{z}\right|<k_{0}$ part of the antenna power spectrum.

(ii) In the case of direct $E_{z}$ excitation due to a misalignment of the FS with the background magnetic field, the lower $\left|k_{z}\right|$ region to be avoided in the power spectrum is enlarged above $k_{0}$.

Consequently, to minimize power losses at the $\mathrm{LH}$, the present analysis suggests having a field aligned FS and to avoid the excitation of the low toroidal part of the antenna power spectrum as in Messiaen et al. (2021). The fact that the power loss is inversely proportional to the derivative of $\epsilon_{1}$ along $x$ also provides a method to directly influence the power loss at the $\mathrm{LH}$ resonance by steepening the plasma density profile near the antenna in the LH density region. The study of Zhang et al. (2019) also points out the potential of using gas puff as a tool to increase the power coupled to the plasma and to further decrease the $\mathrm{LH}$ power loss in ITER provided that the increase of $\left|\epsilon_{1} E_{x}\right|$ does not overcome the one of $\epsilon_{1}^{\prime}$. Those conclusions should also hold for other large fusion devices like DEMO.

While the results are in line with earlier work (e.g. Berro \& Morales 1990; Lawson 1992), the limits of the model should also be emphasized. The model neglects finite temperature effects preventing the detailed description of the wave conversion at the $\mathrm{LH}$ resonance to new electrostatic waves (e.g. ion Berstein waves). The model does not take into account the poloidal and toroidal inhomogeneity of the plasma density profile. It also neglects possible nonlinear effects (e.g. ponderomotive force and sheath effects; a first evaluation of ponderomotive effects is given in Berro \& Morales 1990) arising in the presence of strong $\mathrm{rf}$ fields excited by the antenna. The optimal settings to minimize losses at the LH might not be optimal considering these other phenomena and a trade-off between them might be needed. Finally, the present knowledge of the ITER SOL is derived from theoretical models and therefore there is no guarantee that it corresponds to the experimental density profile in future ITER operations. The model also uses plane 
geometry and an antenna recessed into the wall of the machine with no protruding parts in the far-SOL region.

In conclusion, the phenomena associated with the LH resonance - high fields, surface waves, coaxial modes extending outside the antenna location in the edge region (Messiaen et al. 2021) and, in particular, the related edge power absorption - are possible sources of impurity release that must be taken into account within and on top of the presently considered phenomena such as rf sheath rectification effects and convective cells.

\section{Acknowledgements}

The authors thank Dr L. Colas, CEA, IRFM, F-13108 St-Paul-Lez-Durance, France, for his very helpful input to the paper.

The views and opinions expressed herein do not necessarily reflect those of the European Commission.

Editor Tünde Fülöp thanks the referees for their advice in evaluating this article.

\section{Declaration of interest}

The authors report no conflict of interest.

\section{Funding}

V.M. carried out this work within the framework of the EUROfusion Consortium and has received funding from the Euratom research and training programme 2014-2018 and 2019-2020 under grant agreement No 633053.

\section{Appendix A. A theorem about matrix differential equations}

Theorem A.1. Consider a first-order matrix ordinary differential equation of the form

$$
\frac{d}{d t} x(t)=A(t) x(t)
$$

with $x$ a $n \times 1$ vector and $A$ a $n \times n$ matrix. If $A(t)$ commutes with its integral $\int^{t} A(s) \mathrm{d} s$ then the general solution of the differential equation is

$$
\boldsymbol{x}(t)=e^{\int^{t} A(s) \mathrm{d} s} \boldsymbol{c}
$$

where $c$ is an $n \times 1$ constant vector.

Proof. Using the definition of the matrix exponential, the solution (A 2) can be written

$$
\boldsymbol{x}(t)=\mathrm{e}^{\int^{t} A(s) \mathrm{d} s} \boldsymbol{c}=\sum_{n=0}^{\infty} \frac{\left(\int^{t} A(s) \mathrm{d} s\right)^{n}}{n !} \boldsymbol{c}
$$


Its derivative reads

$$
\begin{aligned}
\frac{\mathrm{d}}{\mathrm{d} t} \boldsymbol{x}(t) & =\frac{\mathrm{d}}{\mathrm{d} t}\left(\sum_{n=0}^{+\infty} \frac{\left(\int^{t} A(s) \mathrm{d} s\right)^{n}}{n !}\right) \boldsymbol{c}, \\
& =A(t) \sum_{n=1}^{+\infty} \frac{\left(\int^{t} A(s) \mathrm{d} s\right)^{n-1}}{(n-1) !} \boldsymbol{c}, \\
& =A(t) \mathrm{e}^{\int^{t} A(s) \mathrm{d} s},
\end{aligned}
$$

where the second equality follows from the fact that, if $\int^{t} A(s) \mathrm{d} s$ commutes with its $t$ derivative $A(t)$, one can write

$$
\frac{\mathrm{d}}{\mathrm{d} t}\left(\int^{t} \boldsymbol{A}(s) \mathrm{d} s\right)^{n}=n A(t)\left(\int^{t} \boldsymbol{A}(s) \mathrm{d} s\right)^{n-1}
$$

We finally have

$$
\dot{x}(t)=A(t) x(t)
$$

\section{Appendix B. Proof of (2.25)}

We will here provide a proof of the identity

$$
\left.\operatorname{Im}[\alpha(x)]\right|_{x=-\kappa} ^{x=+\kappa} \simeq-\frac{\pi}{k_{0}^{2}\left|\epsilon_{1}^{\prime}\right|}
$$

To prove this assertion, one has to notice that, in fact, the LH resonance is not exactly located at $x=0$. Because of the collisions arising in the plasma, the frequency $\omega$ is not real but possesses a small, positive, imaginary part

$$
\omega=\operatorname{Re} \omega+\mathrm{i} \operatorname{Im} \omega, \quad|\operatorname{Im} \omega| \ll|\operatorname{Re} \omega| \quad \text { and } \quad \operatorname{Im} \omega>0 .
$$

Consequently, $\epsilon_{1}$ can be expanded as

$$
\epsilon_{1}(\omega)=\epsilon_{1}(\operatorname{Re} \omega+\mathrm{i} \operatorname{Im} \omega)=\epsilon_{1}(\operatorname{Re} \omega)+\left.\mathrm{i} \operatorname{Im} \omega \frac{\partial \epsilon_{1}}{\partial \omega}\right|_{\omega=\operatorname{Re} \omega}+\mathcal{O}\left((\operatorname{Im} \omega)^{2}\right),
$$

and also exhibits a small imaginary part, $\operatorname{Im} \epsilon_{1} \simeq \operatorname{Im} \omega\left(\partial \epsilon_{1} / \partial \omega\right)$. Recalling ourselves that $\epsilon_{1}(\omega)=1-\sum_{\alpha}\left(\omega_{p \alpha}^{2} /\left(\omega^{2}-\omega_{c \alpha}^{2}\right)\right)$, one can show that $\operatorname{Im} \epsilon_{1}$ is indeed positive

$$
\operatorname{Im} \epsilon_{1} \simeq \operatorname{Im} \omega \frac{\partial \epsilon_{1}}{\partial \omega} \simeq 2 \operatorname{Re} \omega \operatorname{Im} \omega \frac{\partial \epsilon_{1}}{\partial \omega^{2}} \simeq 2 \operatorname{Re} \omega \operatorname{Im} \omega \sum_{\alpha} \frac{\omega_{p \alpha}^{2}}{\left[(\operatorname{Re} \omega)^{2}-\omega_{c \alpha}^{2}\right]^{2}}>0
$$

In the following, we will simply write $\omega$ instead of $\operatorname{Re} \omega$. The main consequence of the discussion above is that $\epsilon_{1}(x)$ does not vanish anymore at $x=0$, but at $x=\bar{x} \equiv$ 
$-\mathrm{i} \operatorname{Im} \epsilon_{1} / \epsilon_{1}^{\prime}$. In other words, the LH resonance is no longer located at $x=0$, but at $x-\bar{x}=0$. This fact is easily implemented in (2.24) by replacing $\left.\operatorname{Im} \alpha(x)\right|_{x=-\kappa} ^{x=+\kappa}$ by

$$
\begin{aligned}
\left.\operatorname{Im} \alpha(x)\right|_{x-\bar{x}=-\kappa} ^{x-\bar{x}=+\kappa} & =\frac{1}{k_{0}^{2}} \operatorname{Im}\left[\frac{\log (\kappa+\bar{x})-\log (-\kappa-\bar{x})}{\epsilon_{1}^{\prime}}\right] \\
& \simeq \frac{1}{k_{0}^{2}} \frac{\arg (\kappa+\bar{x})-\arg (-\kappa-\bar{x})}{\epsilon_{1}^{\prime}} .
\end{aligned}
$$

One has

$$
\begin{aligned}
\arg (\kappa+\bar{x}) & =\arctan \left(\frac{\operatorname{Im}\left(\epsilon_{1}\right)}{\kappa \epsilon_{1}}\right), \\
\arg (-\kappa-\bar{x}) & =\left\{\begin{array}{ll}
\pi-\arctan \left(\frac{\operatorname{Im}\left(\epsilon_{1}\right)}{\kappa \epsilon_{1}}\right), & \epsilon_{1}^{\prime}>0 \\
-\pi+\arctan \left(\frac{\operatorname{Im}\left(\epsilon_{1}\right)}{\kappa \epsilon_{1}}\right), & \epsilon_{1}^{\prime}<0
\end{array} .\right.
\end{aligned}
$$

Noticing that, for $|\kappa| \gg\left|\operatorname{Im} \epsilon_{1} / \epsilon_{1}^{\prime}\right|$, one has

$$
\begin{aligned}
\arg (\kappa+\bar{x}) & \simeq 0, \\
\arg (-\kappa-\bar{x}) & \simeq\left\{\begin{array}{ll}
\pi, & \epsilon_{1}^{\prime}>0 \\
-\pi, & \epsilon_{1}^{\prime}<0
\end{array} .\right.
\end{aligned}
$$

Equation (B 6) becomes

$$
\left.\operatorname{Im} \alpha(x)\right|_{x-\bar{x}=-\kappa} ^{x-\bar{x}=+\kappa} \simeq-\frac{\pi}{k_{0}^{2}\left|\epsilon_{1}^{\prime}\right|}
$$

which is the desired result.

\section{REFERENCES}

Alava, M.J. \& HeikKinen, J.A. 1992 Mode conversion in the vicinity of a fast wave antenna in ICRF-heating. Plasma Phys. Control. Fusion 34 (6), 957.

BELLAN, P.M. 1994 Alfvén 'resonance' reconsidered: Exact equations for wave propagation across a cold inhomogeneous plasma. Phys. Plasmas 1 (11), 3523-3541.

Bellan, P.M. \& PORKOLAB, M. 1974 Propagation and mode conversion of lower-hybrid waves generated by a finite source. Phys. Fluids 17 (8), 1592-1601.

Berro, E.A. \& Morales, G.J. 1990 Excitation of the lower-hybrid resonance at the plasma edge by ICRF couplers. IEEE Trans. Plasma Sci. 18 (1), 142-148.

BoвKOV, V., et al. 2016 Making ICRF power compatible with a high-Z wall in ASDEX upgrade. Plasma Phys. Control. Fusion 59 (1), 014022.

Carpentier, S., Pitts, R.A., Stangeby, P.C., Elder, J.D., Kukushin, A.S., Lisgo, S., FUndAMENSKI, W. \& Moulton, D. 2011 Modelling of beryllium erosion-redeposition on iter first wall panels. J. Nucl. Mater. 415 (1, Supplement), S165-S169. Proceedings of the 19th International Conference on Plasma-Surface Interactions in Controlled Fusion.

DoLGOPOLOV, V.V. 1966 Electromagnetic field singularities in an inhomogeneous magnetoactive plasma (behavior of electric field of expanding wave in magnetically active plasma). Sov. Phys.-Tech. Phys. 11, 198-202.

FAulconeR, D.W. \& Koch, R. 1994 Parasitic absorption at the Alfvén resonance in scrape-off layers. 21st EPS Conf. Control. Fusion Plasma Phys. 18b (II), 1036. 
Golant, V.E. \& PiliYA, A.D. 1972 Linear transformation and absorption of waves in a plasma. Sov. Phys. Uspekhi 14 (4), 413.

Gormezano, C. 1986 Review of lower hybrid wave heating and current drive. Plasma Phys. Control. Fusion 28 (9A), 1365.

HEIKKINEN, J.A. \& BURES, M. 1990 Fast wave antenna coupling to slow waves and ion bernstein waves during ICRF heating of tokamak plasmas. Plasma Phys. Control. Fusion 32 (3), 173.

HoOKe, W.M. \& BERNABEI, S. 1972 Direct observation of waves propagating near the lower-hybrid-resonance frequency. Phys. Rev. Lett. 28 (7), 407.

Kohno, H., MYRA, J.R. \& D'IPPOLITO, D.A. 2015 Numerical investigation of fast-wave propagation and radio-frequency sheath interaction with a shaped tokamak wall. Phys. Plasmas 22 (7), 072504.

Lamalle, P., Beaumont, B., Kazarian, F., Gassmann, T., Agarici, G., Ajesh, P., Alonzo, T., ARAmbhadiYa, B., ARgoUarCH, A., et al. 2013 Status of the ITER ion cyclotron H\&CD system. Fusion Engng Des. 88 (6), 517-520. Proceedings of the 27th Symposium On Fusion Technology (SOFT-27); Liége, Belgium, September 24-28, 2012.

LAWSON, W.S. 1992 Coaxial and surface modes in tokamaks in the complete cold-plasma limit. Plasma Phys. Control. Fusion 34 (2), 175-189.

Lu, L., Crombé, K., Van Eester, D., Colas, L., Jacquot, J. \& Heuraux, S. 2016 Ion cyclotron wave coupling in the magnetized plasma edge of tokamaks: impact of a finite, inhomogeneous density inside the antenna box. Plasma Phys. Control. Fusion 58 (5), 055001.

Maquet, V. \& Messiaen, A. 2020 Optimized phasing conditions to avoid edge mode excitation by ICRH antennas. J. Plasma Phys. 86 (6), 855860601.

Messiaen, A., Koch, R., Weynants, R.R., Dumortier, P., Louche, F., Maggiora, R. \& MILANESIO, D. 2010 Performance of the ITER ICRH system as expected from TOPICA and ANTITER II modelling. Nucl. Fusion 50 (2), 025026.

Messiaen, A. \& Maquet, V. 2020 Coaxial and surface mode excitation by an ICRF antenna in large machines like DEMO and ITER. Nucl. Fusion 60 (7), 076014.

Messiaen, A., Maquet, V. \& Ongena, J. 2021 ICRH fast and slow wave excitation and power deposition in edge plasmas with application to ITER. Plasma Phys. Control. Fusion 63, 045021

Messiaen, A. \& WeYNANTS, R. 2011 ICRH antenna coupling physics and optimum plasma edge density profile. Application to iter. Plasma Phys. Control. Fusion 53 (8), 085020.

Nicolopoulos, A., Campos-Pinto, M. \& Després, B. 2019 A stable formulation of resonant maxwell's equations in cold plasma. J. Comput. Appl. Maths 362, 185-204.

Otin, R., Tierens, W., Parra, F., Aria, S., Lerche, E., Jacquet, P., Monakhov, I., Dumortier, P. \& COMPernolle, B.V. 2020 Full wave simulation of RF waves in cold plasma with the stabilized open-source finite element tool ermes. AIP Conf. Proc. 2254 (1), 050009.

Porkolab, M. 1984 Survey of lower hybrid experiments. IEEE Trans. Plasma Sci. 12 (2), 107-117.

STIX, T.H. 1965 Radiation and absorption via mode conversion in an inhomogeneous collision-free plasma. Phys. Rev. Lett. 15 (23), 878.

STIX, T.H. 1992 Waves in Plasmas. American Institute of Physics.

SWAnson, D.G. 2012 Plasma Waves. Elsevier.

Tierens, W., López, G.S., Otin, R., Urbanczyk, G., Colas, L., Bilato, R., Zhang, W., Bobkov, V. \& Noterdaeme, J.-M. 2020Recent improvements to the ICRF antenna coupling code 'RAPLICASOL'. In AIP Conference Proceedings, vol. 2254, p. 070005. AIP Publishing LLC.

Usoltceva, M., Ochoukov, R., Tierens, W., Kostic, A., Crombé, K., Heuraux, S. \& NoterdaEME, J.M. 2019 Simulation of the ion cyclotron range of frequencies slow wave and the lower hybrid resonance in 3D in RAPLICASOL. Plasma Phys. Control. Fusion 61 (11), 115011.

Zhang, W., Bilato, R., Lunt, T., Messiaen, A., Pitts, R.A., Lisgo, S., Bonnin, X., Bob Kov, V., Coster, D., Feng, Y., Jacquet, P. \& Noterdaeme, J.M. 2019 Scrape-off layer density tailoring with local gas puffing to maximize ICRF power coupling in ITER. Nucl. Mater. Energy 19, 364-371. 